\title{
Impacto del uso de artes de pesca tradicionales en la franja costera de Bilwi
}

\author{
Impact on the use of traditional fishing practices in the coastal strip from Bilwi
}

\author{
Carlos Downs Coleman' \\ Kaleth Robert Dino Steven ${ }^{2}$
}

\section{Resumen}

El presente estudio trata sobre el impacto que genera el uso de las artes de pesca en las zonas costeras del barrio el Muelle y la comunidad El Cocal, de la ciudad de Bilwi. La pesca tradicional se ha convertido en el medio de vida más importante para las familias que han habitado en la franja costera de Bilwi.

Con el pasar del tiempo las artes de pescas comenzaron a innovarse o a mejorar, con la obtención de nuevos materiales y nuevas formas de construcción para mayor aprovechamiento del recurso causando daño al fondo marino y a las especies marinas, provocando impacto de forma negativa a los ecosistemas marinos y hábitats.

Con esta temática se identifica de manera concisa, el impacto provocado por el uso de las artes de pesca, la importancia y el beneficio que genera en cuanto al conocimiento que adquieran los pescadores artesanales. El estudio investigativo es cualitativo a un nivel descriptivo. Se basa en observaciones de las actividades pesqueras que realizan los pescadores, exploración del área de trabajo donde realizan sus actividades pesqueras y con los tipos de artes de pesca con la que capturan. Se describe de manera concisa, los problemas encontrados en el área, de acuerdo a los objetivos de la investigación.

La información para realizar este estudio se obtuvo a través de documentos e investigaciones realizadas por personas intelectuales, entrevistas a autoridades de barrios y a pescadores. Los datos indican que tanto la población pesquera como el esfuerzo pesquero ha venido en aumento, creando condiciones no favorables tal como es la extinción de especies.

Palabras clave: Artes de pesca; ecosistema marino; hábitat; esfuerzo pesquero.

\section{Abstract}

The present study deals with the impact generated by the use of fishing practices in the coastal areas from El Muelle neighborhood and El Cocal community, in Bilwi City. Traditional fishing has become the most important livelihood for families that have inhabited the coastal strip of Bilwi.

With the passing of time the fishing practice began to innovate or improve, with the obtaining of new materials and new forms of construction for greater use of the resource causing damage to the seabed and marine species, causing also negative impact on marine ecosystems and habitats. With this research, the impact caused by the use of fishing gear, the importance and the benefit it generates in terms of the knowledge acquired by artisanal fishermen is concisely identified.

1 Lic. en Pesca, Técnico de IREMADES-URACCAN, correo: rdownscole@yahoo.com, ORCID: http://orcid.org/0000-0002-1913-4063

2 Líder Comunitario Territorio Tawira.

Recibido: 08/08/2019 - Aprobado: 30/08/2019 
The research study is qualitative at a descriptive level. It is based on observations of fishing activities carried out by fishermen, exploration of the work area where they carry out their fishing activities and with the types of fishing practices with which they catch. The problems encountered in the area are described concisely, according to the objectives of the investigation.

The information to carry out this study was obtained through documents and investigations carried out by intellectuals, interviews with neighborhood authorities and fishermen. The data indicates that both the fishing population and the fishing effort have been increasing, creating unfavorable conditions such as the extinction of species.

Keywords: Fishing practices; Marine ecosystem; habitat, fishing effort.

\section{Introducción}

El objetivo principal del presente estudio consistió en el impacto que genera el uso de las artes de pesca en las zonas costeras del barrio el Muelle y la comunidad El Cocal, de la ciudad de Bilwi. Existen muchas familias que se dedican a la actividad pesquera, debido a la falta de empleo que se vive en el municipio, la pesca forma parte del medio de vida de las familias que no tienen empleo y no tienen un ingreso económico, su único medio es la pesca artesanal.

Las artes de pesca que se han estado utilizando en la franja costera han provocado un impacto negativo debido a la forma y método de extracción y aprovechamiento del recurso marino. Las especies más afectadas son las corvinas (Prgyrosamus regius), roncador (Pamadosys incisus), macarela (Decapterus macarellus), palometa (brama brama), camarones (Caridea), y en ocasiones, algunas sardinas (Astyanax aeneus), que se enredan entre las redes. Esta actividad la realizan todo el año, principalmente en las temporadas de reproducción, causando daño a las especies más representativas o comerciales, todo por llevar un sustento económico y alimenticio a sus familiares.

Según González (2002), la actividad pesquera en este municipio proporciona la mayor fuente de trabajo e ingresos económicos a los comunitarios y pobladores asentados en los litorales y se estima que el 90\% de la población depende directa e indirectamente de esta importante actividad.

Desde hace mucho tiempo la pesca se ha venido practicando en las regiones costeras del país, principalmente la Costa Caribe como pesca de subsistencia para el autoconsumo en la familia y lo que sobraba se compartía en la comunidad o bien se utilizaba para el trueque con otros productos, (Velásquez, 2018).

De igual manera, las artes de pescas comenzaron a evolucionar su diseño, así como los procedimientos de empleo de la red, han experimentado una serie de innovaciones y mejoras que las hacen cada día más efectiva (Paramo y Guillot, 2009). Llevar a cabo la regulación de estas artes de pescas, al parecer no es una tarea fácil, puesto que, dentro de las franjas costeras y todo el municipio de Bilwi se encuentra un gran número de familias que se benefician y ven como oportunidad esta actividad.

Según la FAO (2002), la pesca artesanal aportó el 60\% de la actividad pesquera, total, de la región autónoma del atlántico norte, (RAAN), que de casi 3, ooo,ooo de libras, de la producción de pesca, 1, 800,000 libras, son producidas por todas las especies marinas en general, peces, camarones, langostas, cangrejos, es producida por artesanos nativos del lugar, o personas de escasos recursos que se dedican a la pesca.

Debido a la escasa información sobre la captura de descarte en la región, el análisis del presente estudio, es el punto de partida en la valoración de la pesca sobre la comunidad de especies demersales y pelágicos se ha observado la captura de tallas pequeñas de especies con importancia comercial y ecológica. Para poder entender la problemática del impacto de las artes de pesca, es necesario conocer algunas funciones de estas mismas, entendemos por arte de pesca a todas las técnicas que implementan los pescadores, en el caso de la pesca artesanal, los más tradicionales (FAO \& ADPESCA, 2006). 
Durante el estudio se realizaron visitas a las dos zonas costeras más conocidas de la ciudad de Bilwi. Para recabar la información se tuvo que entrevistar en los sitios donde los pescadores realizan su faena de pesca, es decir en la playa, en sus casas. La duración del estudio fue de 15 días consecutivos. Hubo momentos que no se podía tener acceso al sitio por factores climatológico y el otro factor muy relevante por cierto que vale la pena mencionar es que la mayoría de los pescadores pedían a cambio un reconocimiento para poder brindar la información requerida, lo que limito hasta cierta medida la información a fondo.

Este estudio se realizó debido a la importancia que tienen los efectos directos e indirectos de la proliferación de artes de pesca en la zona de los litorales del Caribe Norte. Hasta la fecha no hay estudios basados o relacionados con las diferentes artes de pesca que se utilizan y como las utilizan y el efecto de cada uno de ellos. Se tiene información a manera general, según los comentarios de los pescadores que existen especies de peces que ya no se ven y el volumen de captura ya no es igual a antes.

Esto explica que el número de pescadores aumento de manera rápida, de igual manera el número de apero de pesca, si ante se pescaba con un solo trasmallo se capturaba lo necesario para la subsistencia, pero hoy en día necesitan una mayor cantidad de aperos para poder obtener una producción para su subsistencia. El resultado de esta situación es el declive de la producción en comparación con años anteriores.

\section{Revisión de literatura}

La actividad de la pesca ha sido ejercida desde los albores del desarrollo de la humanidad como una de las actividades fundamentales para conseguir alimento, y para su auto sustento. Para la captura se comenzó por métodos rudimentarios, como los arpones fabricados con ramas de árboles, troncos, anzuelos, ramas que se atravesaban en las zonas poco profundas, las cuales evolucionaron hasta la actualidad que se realiza utilizando una serie de materiales perfeccionados como: la red, elemento de la pesca que está ligado a la historia de la civilización de todos los pueblos, incluso de aquellos que no han tenido relación alguna entre sí (Garcia, 2016).

A los instrumentos y procedimientos que utilizan para capturar a los organismos que pueblan las aguas del planeta, ya sean marinas, salobres o dulces, se les llama, en conjunto, artes y métodos de pesca, aunque por lo general se acostumbra diferenciar, de manera más específica, a la pesca con redes, denominada genéricamente arte, de la que se lleva a cabo por medio de anzuelos y otros aparatos especiales, llamados "aparejos". (Garcia, 2016).

En la actualidad, los modelos de las redes pesqueras son numerosas: unas operan en la superficie, algunas a medias aguas, otras al fondo; sin embargo, todas ellas tienen en común una serie de elementos fundamentales, como los paños, la armadura exterior, los extremos laterales y los cabos (Mora, 2017).

Debido a la escasa información sobre la captura de descarte en la región, el análisis del presente estudio, es el punto de partida en la valoración de la pesca sobre la comunidad demersal costero, como afectan estas artes de pesca caracterizado por captura de tallas pequeñas de especies con importancia comercial y ecológica. De igual manera el cómo se afecta con el aumento de la población pesquera y la cantidad de redes que pescan para las temporadas de pesca en los litorales de la Costa Caribe de Nicaragua.

Para poder entender la problemática del impacto de las artes de pesca, es necesario conocer algunas funciones de estas mismas, entendemos por arte de pesca a todas las técnicas que implementan los pescadores, en el caso de la pesca artesanal, los más tradicionales. (FAO \& ADPESCA, Crecimiento del perfil de la pesca artesanal en las politicas d Nicaragua, 2006), las artes de pescas generalmente se clasifican en dos, activos y pasivos, las cuales se basan en el comportamiento relativo de la especie objeto de la pesca y del arte de pesca. 
Existen varios tipos de artes de pescas diferentes, en este mismo estudio, tenemos algunos clasificados por la FAO \& ADPESCA (2006), en el caso de las redes, tenemos la agallera o red de enmalle, su nombre deriva por el tipo de captura que realiza, ya que los peces suelen ser atrapados por las agallas, específicamente lo enreda.

La pesca de arrastre es una técnica que consiste en el empleo de una red lastrada que barre el fondo del mar capturando todo lo que se encuentre a su paso, sin hacer distinciones. Esta técnica no es selectiva, por lo que se producen capturas accidentales que no resultan útiles en el momento de capturarlas y esto provoca numerosos daños en el medio marino. Es uno de los métodos extractivos de los recursos vivos más utilizados en todo el mundo, pero a la vez, también una de las principales causas de la degradación de los fondos marinos. Se debe de garantizar una explotación sostenible y disminuir las capturas accidentales.

En Nicaragua todavía no se ha hecho realidad la prohibición del uso, la captura de las especies con redes de arrastre costero, pero lo que sí se puede apreciar, que la cantidad de estas artes o métodos de pesca se está proliferando de manera rápida. Aquí en el municipio de Puerto Cabezas se da este fenómeno, debido a los escases de fuentes de empleo y el aumento de la población buscando la manera de como subsistir.

\section{Materiales y métodos}

El presente estudio se realizó desde un contexto o filosofía ancestral, y es que las comunidades son dueños de sus recursos y las riquezas marinas encontradas en un área determinada, se debe de aprovechar al máximo, no importa la cantidad de aperos de pesca y el número de pescadores dedicados a la actividad. No se ha cuantificado exactamente el volumen de descarte producto de la actividad pesquera que se realiza en la zona costera en temporada de mayor actividad de extracción, pero está claro que en la zona costera como foco del presente este estudio, se llenan de basura producto de la actividad de pesca.

Se ha dicho del aprovechamiento de la basura o descarte, transformarla en alimento para aves de corral y otros usos. El estudio básicamente hace énfasis en los efectos que causan los chinchorros y los trasmallos, el número de familia dedicado a esta actividad, la especie que más se captura y el tipo de medio que utilizan para realizar su faena diaria de pesca.

Para la recolección de la información se tuvo que hacer una visita in situ con la participación de la población pesquera nativa, tanto a mujeres, hombres niños y ancianos como parte integral de esta actividad y las visitas de casa en casa. Se diseñó una guía de entrevista la cual estaba diseñada de acuerdo a los objetivos plantados para el estudio. En esta fase de la investigación se estableció una comunicación abierta y directa con los actores del estudio. El lenguaje utilizado en las entrevistas se realizó en su lengua materna (miskitu).

\section{Resultados y discusión}

\section{Comunidad El Cocal}

A partir de las entrevistas formuladas, realizadas y analizadas de cada uno de los barrios de la ciudad de Bilwi, que se dedican a la actividad de pesca, se pudo constatar que los planteamientos hechos son una realidad y que responden a las demandas, necesidades y la realidad en el que viven estos hombres, mujeres y niños que día a día tienen que lidiar para su sostén diario. Es una realidad que los pescadores salen a faenar en sus medios de transporte y medios de extracción de los recursos pesqueros, que les da el sustento diario sin importar las condiciones climatológicas adversas.

Las personas entrevistadas aducen que, como pescadores, la pesca es todo lo que tienen, la pesca es su vida, su negocio. Los pescadores más jóvenes entrevistados, relataron que ellos se dedican a la pesca 
desde la edad de 10 a 11 años, cuando acompañaban a sus familiares y vecinos a pescar y a lanzar redes para conseguir pescado y alimentarse y llevarle a la familia, "es algo que llevamos en la sangre", "ya estamos acostumbrados", "la pesca es mi trabajo de joven y hasta la fecha, obtengo dinero y alimento, es mi vida". Según cuentan anécdotas que ellos aprendieron a pescar o a lanzar redes a través de sus padres que también realizaban este tipo de pesca, uno de ellos dijo que él "aprendió más ayudando a otras personas".

En el caso de las familias que habitan en la comunidad el Cocal no saben cuál es el número exacto de familias que habitan en la en la comunidad, pero "si podemos decir que antes no habia muchas familias viviendo en la comunidad, con el pasar del tiempo, comenzaron a llegar más familias para asentarse en este sector", según lo comentado. Los meses más buenos para pescar son los meses de julio hasta diciembre, porque comienzan a salir más peces, pero por factor tiempo "siempre es peligroso salir, sobre todo en invierno, cuando la marea sube, no se puede salir a pescar porque es muy peligroso".

En la actualidad hay una cifra no precisada, pero si se puede decir que son 35 embarcaciones, de las cuales son 24 cayucos, 5 lanchas y 3 duritara, las que son utilizadas para faenar.

Las especies más comunes que se capturan están las macarelas, camarón, pargo rojo, palometa, corvina, roncador, y otras faunas acompañantes que vienen en los lances que realizan. Las sardinas barbudas, son considerados como fauna acompañante y son aprovechados por los habitantes de esta comunidad o barrio. Para jalar el chinchorro se requieren 6 hombres quienes son los que tienen la tarea de montar la operación en cada lance.

Se tuvieron algunas expresiones:

Honestamente, varias veces he intentado trabajar en otra actividad, pero son trabajos temporales porque después tenemos que dejar por mala paga o porque son trabajos por contrato, después de eso tengo que volver a la casa para seguir dedicándome la pesca, ayudando a mi familia para conseguir dinero, comento un pescador.

El producto de la pesca, por lo general lo comercializan en los mercados locales, o se vende en la comunidad a las familias que habitan dentro de la comunidad el Cocal, para su dieta diaria.

La actividad de la pesca en toda la franja costera se ha venido mermando de manera paulatina y aducen que eso se debe al aumento de la cantidad de aperos de pesca existente y la forma en que estos usuarios le dan uso inapropiado toda la franja costera.

Mediante la entrevista, manifestaron: Los pescadores no tienen un horario ni un reglamento para salir a pescar, todos salimos a pescar a la hora que queremos sin ningún problema, eso ya es de preferencia propia y decisión de cada uno, entendemos que cada quien necesita conseguir algo de alimento y de dinero para sus familiares por eso todos tenemos esa libertad de pescar.

En la zona pesquera o comunidad, comúnmente utilizan las artes de pescas de chinchorro, anzuelo, trasmallo, plomo y red de enmalle, que son los más utilizados. Para ellos estas artes de pesca son las más efectivas para realizar la pesca dentro de la comunidad, ya que se consigue mayor producto. Al producto que no se aprovecha cuando suben el chinchorro se le llama rechazo o basura, lo devuelven al mar ya que estos son especies que no han alcanzado su tamaño comercial o porque no se pueden comer y son aprovechados por personas y aves de rapiña. En casi todos los casos tiran esta basura en la playa contaminando el medio ambiente y el agua, causa posible para que muchas especies se alejen de la franja costera, las especies de mayor valor comercial. 
Medio Ambiente y Recursos Naturales

\section{Barrio El Muelle}

Según la opinión de los pescadores del barrio el Muelle aducen lo siguiente: "Nosotros como pescadores nativos tenemos una ley que nos ampara, la ley No. 28, ley de autonomía, que nos permite disfrutar libremente de nuestros recursos naturales, por eso no necesitamos otra ley".

Los problemas y planteamientos de los pescadores del Muelle son muy similares a los planteados por los otros, aducen que tienen hijos estudiando y lo poco que sacan es para cubrir los gastos de la casa y la educación de sus hijos.

En el 2007, antes de la llegada del huracán Félix, se realizó un censo en el barrio en el barrio El muelle con la ayuda de INETER, se determinó que para esa fecha había un total de 3836 habitantes en el barrio incluyendo niños, jóvenes y adultos, para un total de 719 familias. Lo que significa que en el año 2007 el número de familias por casa tenía dos familias viviendo en una casa, incluyendo tíos y abuelos.

La jueza de barrio dio un resultado de 11 años después del huracán Félix, la población aumentó de manera considerable con un número de familias de 908 familias en total. Lo que significa que actualmente que los habitantes del barrio han crecido considerablemente, pese a eso, el número de casa también ha crecido, ya que cada quien construye para ellos mismos. A pesar de este crecimiento de población, el número de familias por casa no ha cambiado, lo cual significa que el promedio de familias por casa se mantiene constante desde hace 11 años (2007-2018).

En síntesis, lo que ha pasado en el transcurso de estos últimos 10 años es que la población pesquera ha venido en aumento como referencia, a partir del paso del fenómeno natural que azoto la región norte de la costa caribe de Nicaragua. Con este análisis podemos ver que también los artes de pesca y los medios de transporte que se utiliza para la actividad, también ha venido en crecimiento, lo que nos indica que, entre mayor número de pescadores es mayor el esfuerzo pesquero, dándonos como resultado una mayor explotación de los recursos hídricos aprovechables y los no aprovechables por los usuarios dela actividad.

También tiene que ver que, entre mayor número de lances realizado al día por los pescadores, mayor es la destrucción del hábitat de las especies bentónicas y demersales. Se aclara que tanto en la comunidad del Cocal como en el barrio del Muelle esta actividad es la que ha mantenido a los pescadores y habitantes de los barrios mencionados, pero está claro que de una u otra forma esta actividad daña los ecosistemas costeros y al medio ambiente.

En relación a talleres de capacitación, en temas de medios de vida, buenas prácticas pesqueras, aprovechamiento sostenible de los recursos pesqueros son temas de mucha importancia que solicitan para tener conocimiento de los efectos negativos o nocivos como producto de las pesquerías que se realizan en las franjas costeras del municipio de Bilwi, Puerto Cabezas. Es necesario una campaña de sensibilización y concientización en temas de conservación de los recursos marinos y la aplicación de las leyes que rigen para el aprovechamiento sostenible de las especies que están siendo explotadas en mayor cantidad.

\section{Conclusiones}

En síntesis, las artes de pescas dentro de las zonas costeras de Bilwi, son muy útiles para los pescadores, ya que pueden obtener mayor cantidad de especies marinas tales como el camarón (caridea) y algunas especies de peces que se encuentran en la zona, como en el caso de la corvina (Argyorosamus Regius), macarela (Decapterus Macarellus), palometa (Brama brama), sardinas (Astyanax aeneus) y roncador (Pamadasys incisus), las cuales son las especies que se encuentran más dentro de la zona.

En el caso del chinchorro, normalmente se captura todo tipo de especie, de acuerdo a su uso, en el caso del trasmallo, su captura es únicamente peces tanto de fondo como pelágicos. El chinchorro es un arte 
utilizado por las familias, lo cual permite trabajar en conjunto, como ya se mencionó anteriormente, se emplean un mayor número de personas. Puede ser elaborado de forma casera estos aperos de pesca como el chichorro y el trasmallo por su efectividad y fácil de maniobrar.

Durante el transcurso de esta investigación, se pudo conocer la gran importancia que tienen las artes de pesca para las familias costeñas de Puerto Cabezas, por los beneficios que han brindado al realizar la pesca como único medio de subsistencia. Por esta razón, las familias costeras de Puerto Cabezas han comenzado a utilizar artes de pescas, sobre todo las redes como el chinchorro y el trasmallo. A medida que va pasando el tiempo, los chinchorros y las demás redes van aumentando en cantidad en el municipio, dentro de las zonas costeras, tanto el Cocal como el Muelle. Hoy en día hay muchas familias que tienen chinchorros y trasmallos, los cuales son los más utilizados para salir a pescar.

Debido a lo anterior, se ha generado un problema en cuanto al desarrollo de algunas especies marinas, tal como lo plantea Narváez (2008), sobre el crecimiento de talla del camarón, en el caso de nuestro sector, algunas de las especies afectadas en su desarrollo son la tortuga verde y el roncador, que muchas veces suelen ser capturadas por las redes.

En ocasiones, las especies que son capturadas no han alcanzado su etapa de desarrollo y son tirados a la intemperie como descarte y abandonados en las orillas de las playas, lo cual provoca impacto al medio ambiente, sobre todo perjudicando a las familias que viven cercanas a las playas, provocando el mal olor, mal aspecto, lo que estimula enfermedades a los habitantes, sobre todo a los niños.

Así mismo, el desecho de estas mismas, también atrae aves de rapiña en las zonas, lo que provoca un aspecto desagradable para los pobladores y turistas que llegan al lugar. Todos estos problemas encontrados, son debido al mal uso y mal aprovechamiento de las artes de pesca tradicionales en la región o en estas zonas,

Los testimonios de los pescadores relatan que debido al crecimiento demográfico de las zonas costeras El Muelle y El Cocal, el aumento de las artes de pescas como el chinchorro y el trasmallo, se ha utilizado en gran número cada vez que salen a pescar. Según un dato aproximado, de un total de 12 pescadores que salen a pescar por las mañanas, había aproximadamente 4 chinchorros, y 3 tres trasmallos para pescar, esa es la rutina diaria.

Según el testimonio de los entrevistados algunos pescadores o familias llevan años habitando en la zona y utilizan hasta 4 artes de pesca distintos o la misma red de arrastre como chinchorro o trasmallo, para que dentro de la familia se utilicen por separado, y esto provoca que el impacto sea mayor, que la disminución de especies marinas sea aún más perjudicial.

\section{Agradecimiento}

Esta publicación obtuvo el financiamiento del Proyecto Ecosistemas Costeros, auspiciado por: Unión Europea, Dka Austria, Cooperación Austriaca para el Desarrollo y Horizontzooo.

\section{Lista de referencias}

FAO. (2006). Clasificacion de artes y métodos de pesca trdicionales. veracruz: FishCod-STF.

FAO, \& ADPESCA. (2006). Crecimiento del perfil de la pesca artesanal en las politicas de Nicaragua. Managua: FishCode-STF-WP. 
García, C. (2016). Conocimiento Tradicional: Lo que los pescadores del caribe colombiano tienen para decirnos. bogota: PANAMJAS, pan-american journal of aquatic sciences.

Mora, A. e. (2017). Analisis de la Comunidad de Peces de Descarte en la Pesca de Arrastre del Camaron (temporada de lluvias 2013) en la Zona Central Sur del Litoral Veracruzano, Mexico. Veracruz: ISSN.

Paramo, J., \& Guillot, L. (2009). Aspectos Poblacionales y Ecológicos de Peces Demersales de la Zona Norte del Caribe Colombiano en Relación con el Habitat: una Herramienta Para identificar Áreas Marinas Protegidas (AMPs) para el Manejo Pesquero. Bogota: Caldasia.

Velásquez, E. (2018). Administración de los Recursos Pesqueros de Nicaragua, Organización, Monitoreo, Evaluación y Regulación. Puerto Cabezas, INPESCA central. 